\title{
Analysis and Design of Periodic Leaky-Wave Antennas for the Millimeter Waveband in Hybrid Waveguide-Planar Technology
}

\author{
José Luis Gómez-Tornero, Fernando Daniel Quesada-Pereira, and Alejandro Álvarez-Melcón, Member, IEEE
}

\begin{abstract}
This work presents a full-wave integral equation approach specifically conceived for the analysis and design of laterally-shielded rectangular dielectric waveguides, periodically loaded with planar perturbations of rectangular shape. This type of open periodic waveguide supports the propagation of leaky-wave modes, which can be used to build leaky-wave antennas which exhibit many desirable features for millimeter waveband applications. The particularities of the leaky-mode analysis theory are described in this paper, and comparisons with other methods are presented for validation purposes. Using this leaky-mode analysis method, a novel periodic leaky-wave antenna is presented and designed. This novel antenna shows some important improvements with respect to the features of previously proposed antennas. The results of the designed radiation patterns are validated with three-dimensional electromagnetic analysis using commercial software.
\end{abstract}

Index Terms-Floquet modes analysis, leaky-wave antennas, millimeter waveband, periodic antennas.

\section{INTRODUCTION}

$\mathbf{T}$ HE radiation mechanism of periodic leaky-wave antennas (PLWA) is well-known for many decades [1], [2]. This type of antennas exhibits interesting features due to their traveling-wave nonresonant nature. Among them we can mention frequency scanning capability, high directivity and large radiation bandwidths. Low-losses, easy-manufacturing, open waveguides can be used to conceive PLWA, thus leading to new antennas very well suited for millimeter waveband applications. Different technologies have been used to design PLWA, as the microstrip line [3], [4], the dielectric grating guide [5], the strip-loaded dielectric slab [6]-[9] and the strip-loaded inset waveguide [10]-[12]. Recently, a hybrid planar-waveguide technology, which makes use of a laterally-shielded top-open dielectric rectangular waveguide, in which an uniform strip or slot is asymmetrically located to provide radiation, was proposed to design tapered uniform LWA for the millimeter waveband [13]. The same technology can be used to obtain backward radiation, by changing the waveguide perturbations from uniform to periodic [2], leading to the PLWA in hybrid planar-waveguide technology shown in Fig. 1.

Manuscript received August 12, 2004; revised April 6, 2005. This work was supported by Spanish National projects ESP2001-4546-PE and TEC2004-04313-C02-02/TCM and Regional Séneca projects 2002 PB/4/FS/02 and PMPDI-UPCT-2004.

The authors are with the Departamento de Tecnologías de la Información y las Comunicaciones, Universidad Politécnica de Cartagena, Antiguo Hospital de Marina, 30202 Cartagena (Murcia), Spain (e-mail: josel.gomez@upct.es).

Digital Object Identifier 10.1109/TAP.2005.854562

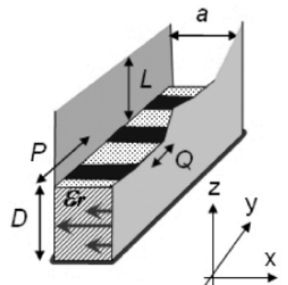

a)

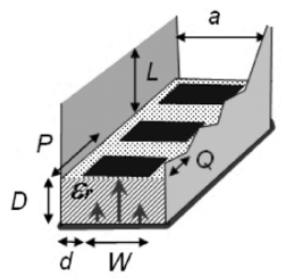

b)

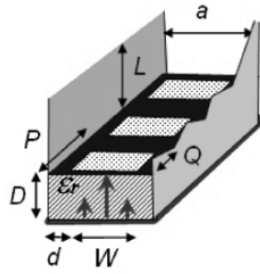

c)
Fig. 1. Different types of laterally-shielded top-open rectangular dielectric guides, periodically perturbed with rectangular planar circuitry. (a) Dielectric-inset PLWA. (b) Strip-Loaded PLWA. (c) Slot-Loaded PLWA.

This type of structures must not be confused with the striploaded dielectric slab PLWA, as those studied in [6]-[9]. The antennas proposed in this work are based on a laterally-shielded rectangular dielectric waveguide, and present a parallel plate stub of height $L$ at their top (see Fig. 1). An analysis method conceived for this type of antennas was developed in [10]-[12]. However, some differences arise between the method proposed in this paper and the techniques used in [10] and [12]. The scattering analysis carried out in [10] did not take into account for the mutual coupling effects between array elements. In [11], Guglielmi and Boccalone proposed a more accurate transverse equivalent network (TEN) to represent the unit cell of the PLWA, which is used to design a novel dielectric inset PLWA in [12]. The method proposed in [11] and [12], although accurate, has two important restrictions which are overcome by the full-wave analysis technique proposed in this paper. The first restriction of [11], [12] is that only one-dimensional (1-D) periodic discontinuities can be studied, leading to the strip loaded PLWA shown in Fig. 1(a) (the dielectric-inset PLWA). The novel PLWA shown in Fig. 1(b) and (c) need the introduction of two-dimensional (2-D) perturbations to obtain interesting taper properties, as it will be shown in Section III of this paper. The second difference is that the TEN developed in [11], [12] is valid only for the $\mathrm{TE}_{01}$ mode excitation [horizontally polarized electric field, as shown in Fig. 1(a)] The new antennas proposed in this paper are based on the excitation of the $\mathrm{TE}_{10}$ mode (vertical polarization), as illustrated in Fig. 1(b) and (c).

In this paper, a novel slot-loaded PLWA [Fig. 1(c)] is presented, showing the main advantages with respect to the dielectric-inset PLWA [Fig. 1(a)]. A full-wave spectral-domain leakymode analysis method is described in Section II of this paper. Comparisons with the results obtained with other techniques for leaky-modes in different laterally-shielded planar structures are included for validation purposes. Section III presents the novel PLWA. The working mechanism is explained, and a tapered an- 
tenna is designed to illustrate the improvements with respect to the dielectric-inset PLWA proposed in [12]. Finally, the radiation patterns of the designed antenna are validated using commercial electromagnetic analysis software (HFSS).

\section{SPECTRAL-DOMAIN LEAKY-MODE ANALYSIS}

The type of LWA to analyze is formed by a multilayered top-open medium in the transverse $z$-direction, which is surrounded by lateral metal walls separated at a distance $a$ in the $x$-direction (see Fig. 1). In the longitudinal $y$-direction of the structure, the antenna is infinite and periodic, with a periodicity $P$ which determines the unit cell length. Applying Floquet theory, the fields can be expanded in the $y$-direction using Floquet modes [2], [4], [5]

$$
\begin{aligned}
\vec{E}(x, y, z) & =e^{-j k_{y 0} y} \cdot \vec{E}_{p}(x, y, z) \\
& =e^{-j k_{y 0} y} \cdot \sum_{m_{Y}=-\infty}^{+\infty} \vec{E}_{m_{Y}}^{P}(x, z) \cdot e^{-j m_{Y} \frac{2 \pi}{P} y} \\
& =\sum_{m_{Y}=-\infty}^{+\infty} \vec{E}_{m_{Y}}^{P}(x, z) \cdot e^{-j k_{y m_{Y}} y} \\
\vec{H}(x, y, z) & =\sum_{m_{Y}=-\infty}^{+\infty} \vec{H}_{m_{Y}}^{P}(x, z) \cdot e^{-j k_{y m_{Y}} y} \\
k_{y_{m_{Y}}} & =k_{y 0}+m_{Y} \frac{2 \pi}{P} ; m_{Y}=-\infty, \ldots,+\infty
\end{aligned}
$$

The index $m_{Y}$ stands for the order of the Floquet modes, while $k_{y 0}$ is the propagation constant of the bloch-wave. Moreover, the electric and magnetic fields in the unit cell, $\vec{E}_{m_{Y}}^{P}(x, z)$ and $\vec{H}_{m_{Y}}^{P}(x, z)$, can also be expanded in the $x$-direction using a series of parallel-plate waveguide (PPW) modes

$$
\begin{aligned}
\vec{E}_{m_{Y}}^{P}(x, z)= & \sum_{m_{X}=0}^{\infty} \sum_{p}^{\mathrm{TE}, \mathrm{TM}} V_{m_{X}, m_{Y}}^{(p)}(z) \cdot \vec{e}_{m_{X}, m_{Y}}^{(p)}(x) \\
& +\frac{1}{j \omega \varepsilon} \cdot \sum_{m_{X}=0}^{\infty} I_{m_{X}, m_{Y}}^{\mathrm{TM}}(z) \\
& \cdot \sqrt{k_{x m_{x}}^{2}+k_{y m_{y}}^{2}} \cdot \phi_{m_{X}, m_{Y}}^{\mathrm{TM}}(x) \cdot \hat{z} \\
\vec{H}_{m_{Y}}^{P}(x, z)= & \sum_{m_{X}=0}^{\infty} \sum_{p}^{\mathrm{TE}, \mathrm{TM}} I_{m_{X}, m_{Y}}^{(p)}(z) \cdot \vec{h}_{m_{X}, m_{Y}}^{(p)}(x) \\
& +\frac{1}{j \omega \mu} \cdot \sum_{m_{X}=0}^{\infty} V_{m_{X}, m_{Y}}^{\mathrm{TE}}(z) \\
& \cdot \sqrt{k_{x m_{x}}^{2}+k_{y m_{y}}^{2}} \cdot \phi_{m_{X}, m_{Y}}^{\mathrm{TE}}(x) \cdot \hat{z} .
\end{aligned}
$$

The analytical expressions of the PPW modal vector-field functions, $\vec{e}_{m_{X} m_{y}}^{(p)}(x), \phi_{m_{X} m_{y}}^{(p)}(x) \cdot \hat{z}$ and $\vec{h}_{m_{X} m_{y}}^{(p)}(x)$, can be found in [14]. The index $p$ distinguishes between $\mathrm{TE}^{\mathrm{Z}}$ and $\mathrm{TM}^{\mathrm{Z}}$ polarizations, while the order of the PPW mode $m_{X}$ establishes the harmonic variation of the fields along the $x$-axis

$$
k_{x m_{X}}=m_{X} \cdot \frac{\pi}{a}(\operatorname{rad} / m), \quad m_{X}=0, \ldots,+\infty
$$

In the transverse $z$-direction, a set of equivalent transmission lines can be obtained for each PPW-Floquet mode combination (index $m=\left\{m_{X}, m_{y}\right\}$ ), following the same procedure applied in [14] for uniform LWA. This equivalent network models the propagation of the fields along the transverse multilayered media, as well as the top radiating open end, whose equivalent radiation impedance $Z_{\mathrm{RADm}}^{(p)}$ can be found in [15]. The equivalent voltage and current functions, $V_{m_{X}, m_{Y}}^{(p)}(z)$ and $I_{m_{X}, m_{Y}}^{(p)}(z)$, can be obtained from these transmission lines, as described in [15]. Following the procedure described in [15], the method of moments (MoM) can be applied to expand the electric currents on the periodic strips (or alternatively, the equivalent magnetic currents on the periodic slots), and to solve the corresponding electric field integral equation (EFIE, or alternatively, a magnetic field integral equation, MFIE). In any case, the Gallerkin procedure is applied, so an homogeneous system of linear equation must be solved for the coefficients of the current expansion in the unit cell. In order to ensure non trivial solution, the determinant of the MoM matrix $M$ must be equal zero, leading to the next well-known eigenvalue equation

$$
\operatorname{det}\{M\}=\Psi\left(k_{y m y}\right)=0 \text {. }
$$

The characterization of PLWA can be carried out by obtaining the complex propagation constant of the leaky-wave modes. Due to the radiation losses, the bloch-wavenumber $k_{y 0}$ becomes complex, having a phase constant $\beta_{y 0}$ and an attenuation constant $\alpha_{y}$. In this way, the propagation constant of any space harmonic $\left[k_{y m y}\right.$ in (3)], can be computed by the next expression

$$
\begin{aligned}
k_{y m_{y}} & =\left(\beta_{y 0}-j \alpha_{y}\right)+m_{y} \frac{2 \pi}{P} \\
& =\left(\beta_{y 0}+m_{y} \frac{2 \pi}{P}\right)-j \alpha_{y}=\beta_{y m_{y}}-j \alpha_{y} .
\end{aligned}
$$

This section presents some comparisons between the results obtained with the proposed method, and other results published in previous works. However, it must be noticed that, to the authors' knowledge, there is not any published technique to deal with the proposed 2-D rectangular periodic perturbations inside a multilayered laterally-shielded open structure. Spectral domain Gallerkin methods have been applied for the study of periodic 2-D planar structures [16], [17], but completely enclosed in a rectangular metallic cover, therefore, not allowing for the existence of leaky-modes. For the case of laterally-shielded top open leaky-wave structures, only 1-D planar perturbations located at the dielectric-air interface have been studied so far. For example, in [18]-[20], different types of uniform LWA were conceived by adding to the original nonradiative guide some kind of nonperiodic planar perturbation in the transverse $x$-axis to induce radiation. In other published works, 1-D periodic strip gratings in the longitudinal $y$-direction of a dielectric slab [6]-[9] or of a dielectric inset guide [10]-[12] [see Fig. 1(a)] were studied. For this reason, the comparisons made in this section do not include any 2-D planar perturbation in the dielectric guide. Nevertheless, these comparisons with results for 1-D LWA can be used to validate the ability of the program to model planar discontinuities in the interface of a laterally-shielded dielectric guide, for both the $x$ and $y$ directions of Fig. 1 . 

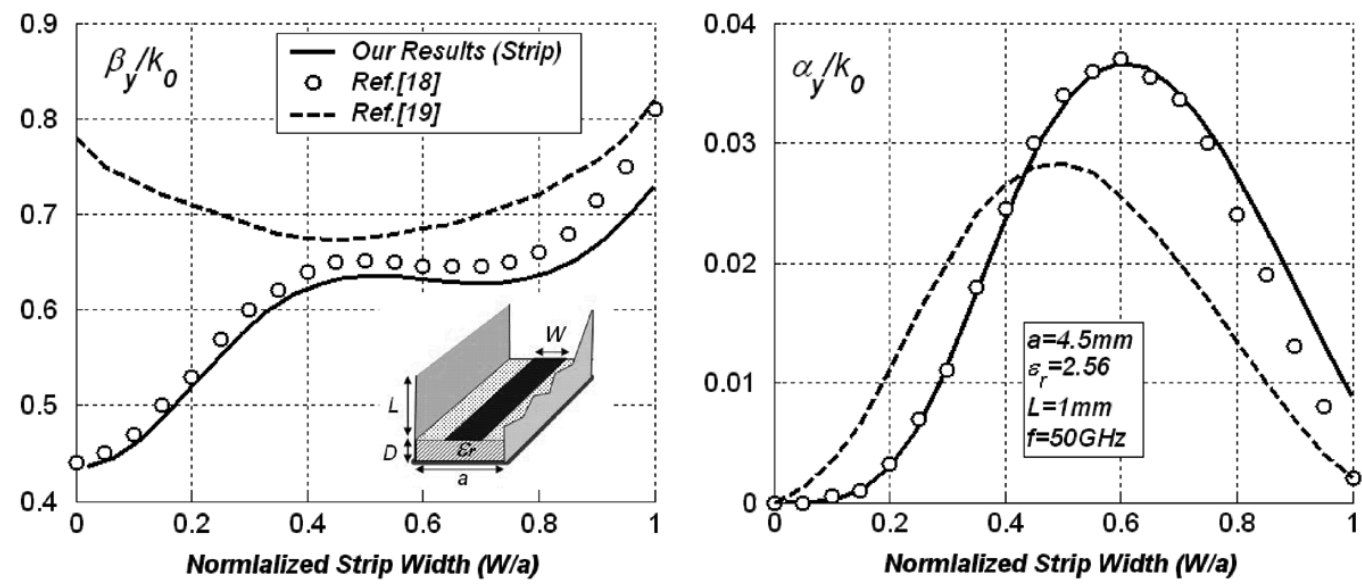

Fig. 2. Comparison of results for 1-D uniform perturbation in the $x$-direction.
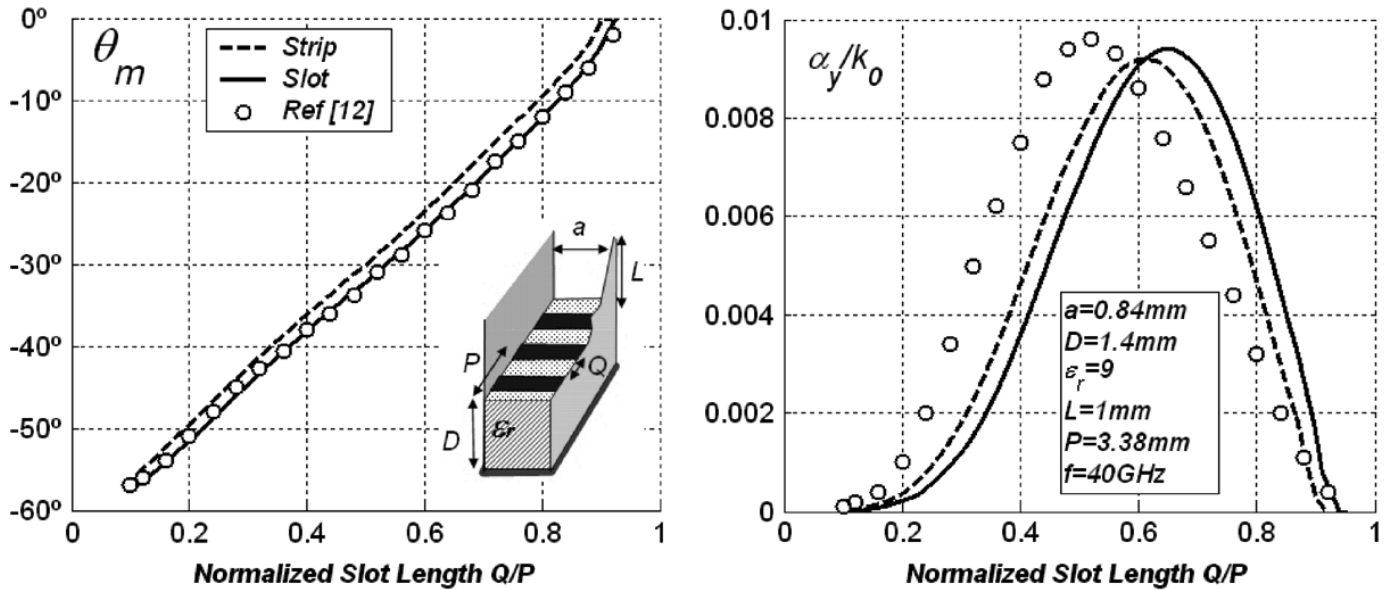

Fig. 3. Comparison of results for 1-D periodic perturbation in the $y$-direction.

To validate the results for the planar discontinuity in the $x$-direction, results for the first higher order mode of a laterally-shielded microstrip line are presented in Fig. 2. The dimensions of the structure are: $a=4.5 \mathrm{~mm}, D=1.59$ $\mathrm{mm}, \varepsilon_{r}=2.56, L=1 \mathrm{~mm}$, and the frequency of analysis is $f=50 \mathrm{GHz}$. Our results are compared to those obtained using a full-wave spectral domain technique [18], and results from a transverse resonance technique [19], [20]. This last technique makes use of a transverse equivalent network (TEN) for the strip discontinuity, which is obtained in closed form for small obstacles. The small obstacle approximation, also used for periodic discontinuities [21], is less valid for large aperture values, as can be seen for the dashed line in Fig. 2, when $W / a<0.4$. Our approach agrees very well with the results obtained with the full-wave spectral domain approach, for both the phase and the attenuation constant of the microstrip higher order leaky-wave mode. $N_{x}=4$ basis functions and $M_{x}=100$ PPW modes were used.

Fig. 3 shows the results obtained for the main leaky-wave mode in the dielectric-inset PLWA shown in Fig. 1(a). This antenna was studied by Guglielmi in [12] using a TEN developed in [11] to model the planar periodic metal strip grating in the dielectric-air interface. The dimensions of the antenna, according to the scheme shown in Fig. 1(a) are: $a=0.84 \mathrm{~mm}$, $\varepsilon_{r}=9, D=1.4 \mathrm{~mm}, L=1 \mathrm{~mm}$, and $P=3.38 \mathrm{~mm}$. Fig. 3 represents the variation of the angle of maximum radiation of the $m_{y}=-1$ space-harmonic $\left(\sin \theta_{m}=\beta_{m y=-1} / k_{0}\right)$ and the normalized attenuation constant $\left(\alpha_{y} / k_{0}\right)$ as the slot aperture length $Q$ is modified. The circles represent the results obtained in [12] with the TEN. The continuous line represent the results obtained with the method proposed in this paper, by expanding the electric currents in the periodic strip. The dashed line represents the results for the slot magnetic currents expansion. Both results agree very well with those obtained in [12]. The number of basis function used are $N_{y}=4$, with a total of 21 Floquet modes $\left(M_{y}=10\right)$.

\section{A NOVEL PLWA}

In this section, a novel PLWA in hybrid printed-circuit dielectric-waveguide technology is presented. This antenna is geometrically quite similar to the dielectric-inset PLWA studied in [12], but has a totally different radiation principle. This fact provides a very important electrical feature which will be shown: it allows to independently control the stopband and the illumination of the antenna. In this section, the working mechanism of the inset PLWA is firstly summarized. Then, the novel PLWA is presented and the radiation mechanism is explained. Some leaky-wave 

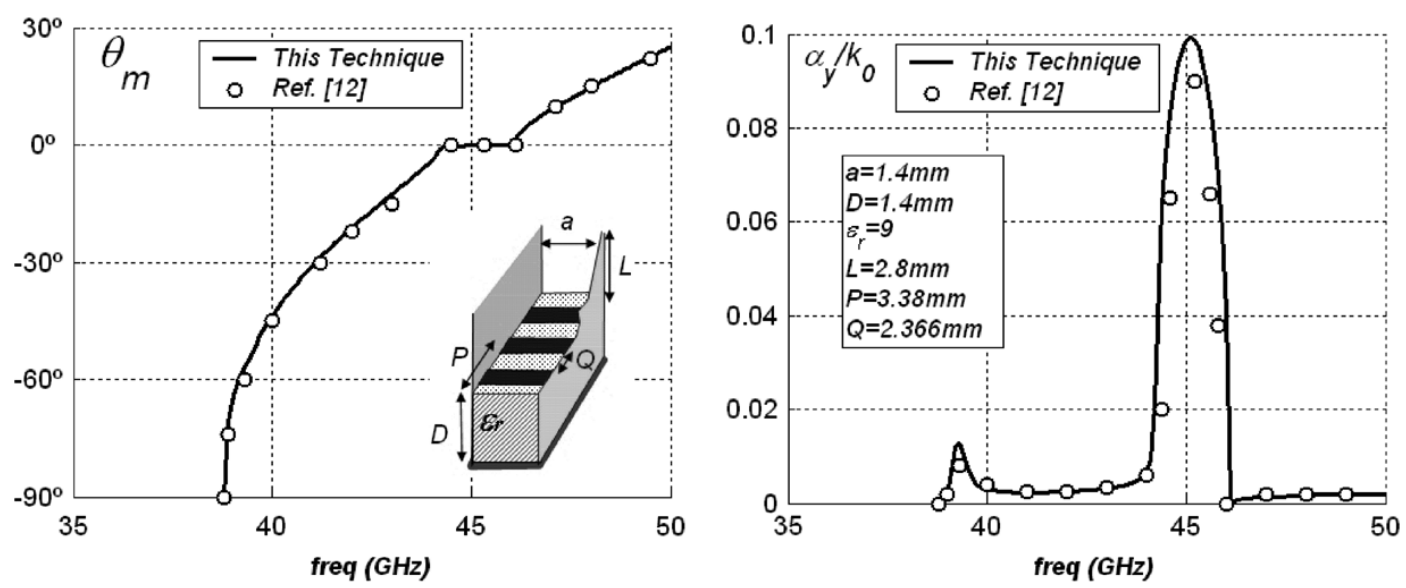

Fig. 4. Backward to forward frequency scanning capability of the dielectric-inset PLWA.

mode dispersion curves are plotted to show the electrical performances of the proposed antenna. Finally, a cosine illuminated PLWA is designed using the novel leaky-mode method of moments analysis. The predicted radiation patterns are compared with results from three-dimensional (3-D) analysis obtained with commercial software (HFSS).

\section{A. Dielectric-Inset PLWA Working Mechanism}

The backward radiation principle of PLWA is well-known [2], [5]. The $m_{y}=-1$ space harmonic is used to obtain backward to forward frequency scanning. Fig. 4 shows the results obtained for the dielectric-inset PLWA designed in [12] (circles), together with the results obtained with the analysis technique developed in this paper (continuous line). Very good agreement between both methods is observed.

As described in [12], the period $P$ is chosen to select the radiation frequency and to avoid multi space-harmonic radiation. On the other hand, the slot length $Q$ can be varied to modify the bandwidth of the bandgap which occurs at broadside. Fig. 5 shows the results obtained in [12] (circles) as compared with those obtained with the proposed analysis technique (continuous line), for several $Q$ values. Again, very good agreement is shown. As it can be seen in Fig. 6, there is a given value of $Q$ which should be chosen to minimize the stopband $(Q=2.028$ $\mathrm{mm}$ ), thus obtaining a more continuous scanning transition from backward to forward radiation. To achieve broadside radiation, a more complicated two-slot structure should be used, as demonstrated in [22].

The illumination of a leaky-wave antenna must be somehow tapered to reduce the sidelobes level. For this purpose, the leakage rate of the leaky-mode must be varied along the antenna length, while keeping unchanged the pointing direction of the antenna [2]. The dielectric-inset PLWA can modify the leakage rate by varying the relative slot length, $Q / P$, as illustrated in Fig. 3. However, two important problems arise from this mechanism to control the leakage rate. First, the slot length controls the stopband (Fig. 5). Therefore, one cannot independently control the bandgap and the illumination of the antenna. Second, the variation of $P / Q$ strongly affects both the pointing direction of the antenna and the leakage rate. As it is well-known [2], this causes phase aberration in the radiation

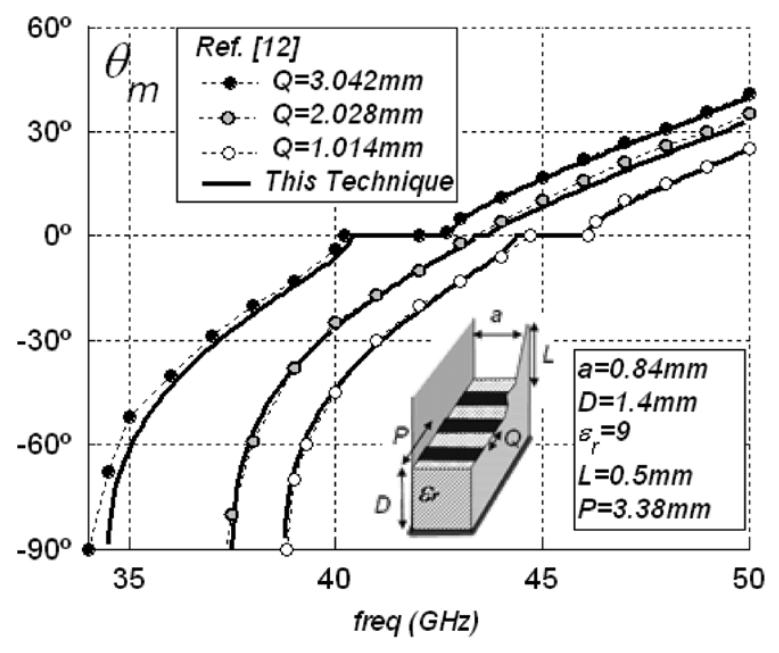

Fig. 5. Control of the bandgap in the dielectric-inset PLWA.

pattern. It is then desirable to independently control $\theta_{m}$ and $\alpha$ to allow for an easier tapering procedure. The proposed PLWA overcome these two difficulties inherent to the dielectric-inset PLWA.

\section{B. The Asymmetrical PLWA}

The proposed PLWA is shown in Fig. 1(c). It is quite similar to the dielectric-inset PLWA, but has two main differences. First, the periodic slots do not cover all the waveguide width $a$, but have a width $W<a$, and are located at an offset $d$ from one side (see Fig. 1). Second, the waveguide must be excited with the $\mathrm{TE}_{10}$ mode, and not with the $\mathrm{TE}_{01}$ mode as it happened in the dielectric-inset PLWA. These two facts make the radiation mechanism of the new antenna to be based on the asymmetry principle, illustrated in Fig. 6. When the periodic slots are located symmetrically with respect to the side walls, the vertically polarized $\mathrm{TE}_{10}$ mode does not create any horizontal field in the antenna aperture, as shown in Fig. 6(a). As it is well-known in this type of stub-loaded LWA [2], [15], [19], [20], the parallel plates act as a filtering mechanism which only allow the horizontal field to reach the waveguide aperture, located at a height $L$ from the dielectric interface. Therefore the symmetrical periodic structure makes the $\mathrm{TE}_{10}$ mode to be nonradiative if the 


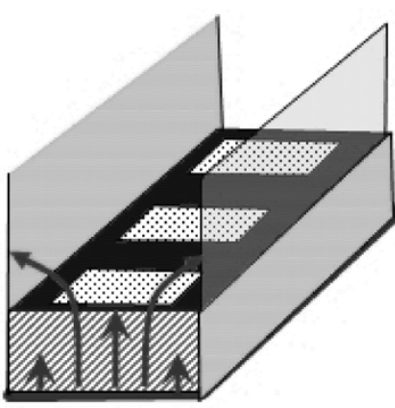

a)

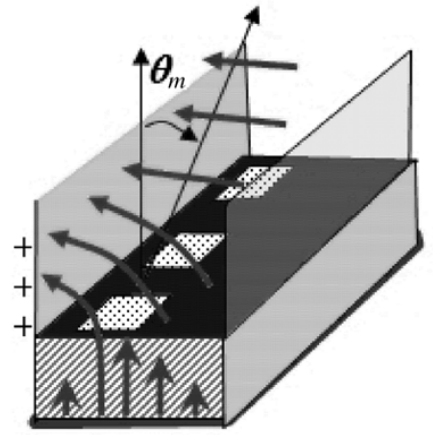

b)

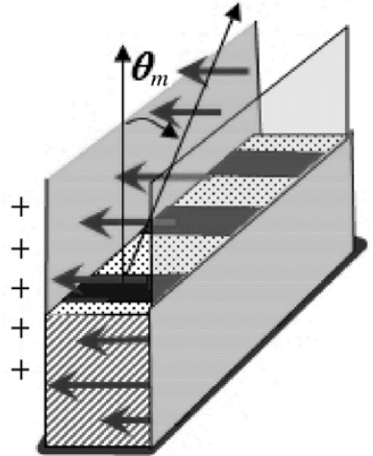

c)

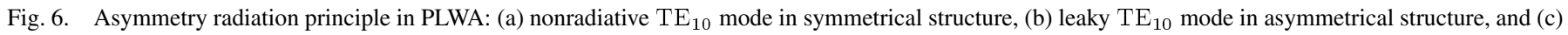
leaky $\mathrm{TE}_{01}$ mode in symmetrical structure.

stub length $L$ is high enough [15]. The slots must be asymmetrically located to induce a difference of potential in the parallel plates. This difference of potential creates a horizontal polarized TEM wave which can propagate to the aperture, and can radiate to the free space region, as it is shown in Fig. 6(b).

Therefore, by controlling the level of asymmetry of the slots, one can control the level of excitation of this radiating horizontal wave, and as a result the leakage rate of the $\mathrm{TE}_{10}$ leakymode can be adjusted. For this reason, the new PLWA will be called asymmetrical PLWA (APLWA). On the contrary, the inset PLWA is based on the excitation of the $\mathrm{TE}_{01}$ mode, which is horizontally polarized itself. The own $\mathrm{TE}_{01}$ mode is responsible for the radiation of the antenna, therefore, there is no need to introduce any asymmetry to induce radiation in the structure, as illustrated in Fig. 6(c).

Nevertheless, the rest of the working principles of the inset PLWA are also applicable to the new APLWA. The period $P$ must be chosen to select the operation frequency, while the value of $Q$ allows to modify the stopband width. Fig. 7 shows the dispersion curves of the $m_{y}=-1$ space harmonic obtained for the new leaky-wave structure with the next dimensions: $a=1.4$ $\mathrm{mm}, D=0.84 \mathrm{~mm}, \varepsilon r=9, L=0.5 \mathrm{~mm}, d=0.3 \mathrm{~mm}$, $W=0.6 \mathrm{~mm}$, and $P=2.8 \mathrm{~mm}$. It must be noticed that the dielectric guide dimensions have been interchanged with respect to the dielectric-inset PLWA ( $a=1.4 \mathrm{~mm}, D=0.84 \mathrm{~mm}$ ), in order to work with the vertically polarized $\mathrm{TE}_{10}$ mode.

In our case, the value of $Q$ which minimizes the stopband is $Q=1 \mathrm{~mm}$. With this value, it is obtained the millimeter waveband frequency-scanning response shown in Fig. 8, where also the variation of the leakage constant with frequency is shown. It can be seen that the response is quite similar to the one obtained for the inset PLWA (Fig. 4), with the difference that the stopband width has been reduced.

As illustrated in Fig. 3, the only way to control the leakage rate in the inset PLWA is by changing the slot aperture $Q$. The main novelty of the new APLWA is that the leakage rate can be controlled by modifying the slot asymmetry, while keeping unchanged the value of $Q$, as previously explained. Fig. 9 illustrates this mechanism, by showing the variation of the pointing direction $\theta_{m}$ and the normalized leakage rate $\alpha / k_{0}$ when the slot width $W$ is modified for different values of the slot offset

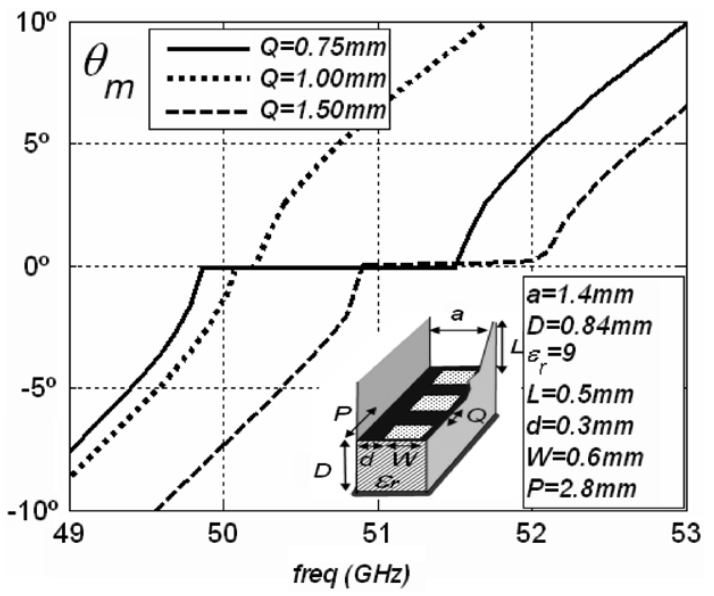

Fig. 7. Control of the bandgap in the novel APLWA.

d. The antenna dimensions are the same of that in Fig. 8, and the frequency of analysis is $45 \mathrm{GHz}$, which corresponds to a pointing angle in the backward quadrant $\left(\theta_{m} \approx-38^{\circ}\right)$. For a given slot offset $d$, there is a value of the slot width which makes the structure to be symmetrical with respect to the lateral parallel-plate walls $(W=a-2 d)$. The leakage rate is zero for these values of the slot width, as it is seen in Fig. 9(b). As the width is decreased, the structure becomes more asymmetrical and the leakage rate increases.

However, it can be seen in Fig. 9(a) that $\theta_{m}$ also suffers a small variation when the slot width is modified. As it is wellknown [2], the pointing direction must be kept unchanged in a tapered leaky-wave antenna to avoid phase aberrations. In order to keep $\theta_{m}$ constant while varying $\alpha / k_{0}$, both the slot width $W$ and the slot offset $d$ must be modified at the same time. As it can be seen in Fig. 9(a), the slot offset $d$ can be modified to readjust the pointing direction, while $W$ is varied to obtain the different values of $\alpha / k_{0}$. As a result, it can be obtained a contour curve with the values of $W$ and $d$ which allow to change $\alpha / k_{0}$ from zero to a given leakage rate, while keeping constant $\theta_{m}$. Fig. 10 shows the constant $\theta_{m}$ curve for the APLWA, with a constant pointing angle of $\theta_{m}=-38^{\circ}$ at $45 \mathrm{GHz}$.

As it can be seen from Fig. 10, one can vary the normalized leakage rate from zero to $\alpha / k_{0}=0.0015$. The periodic slot 

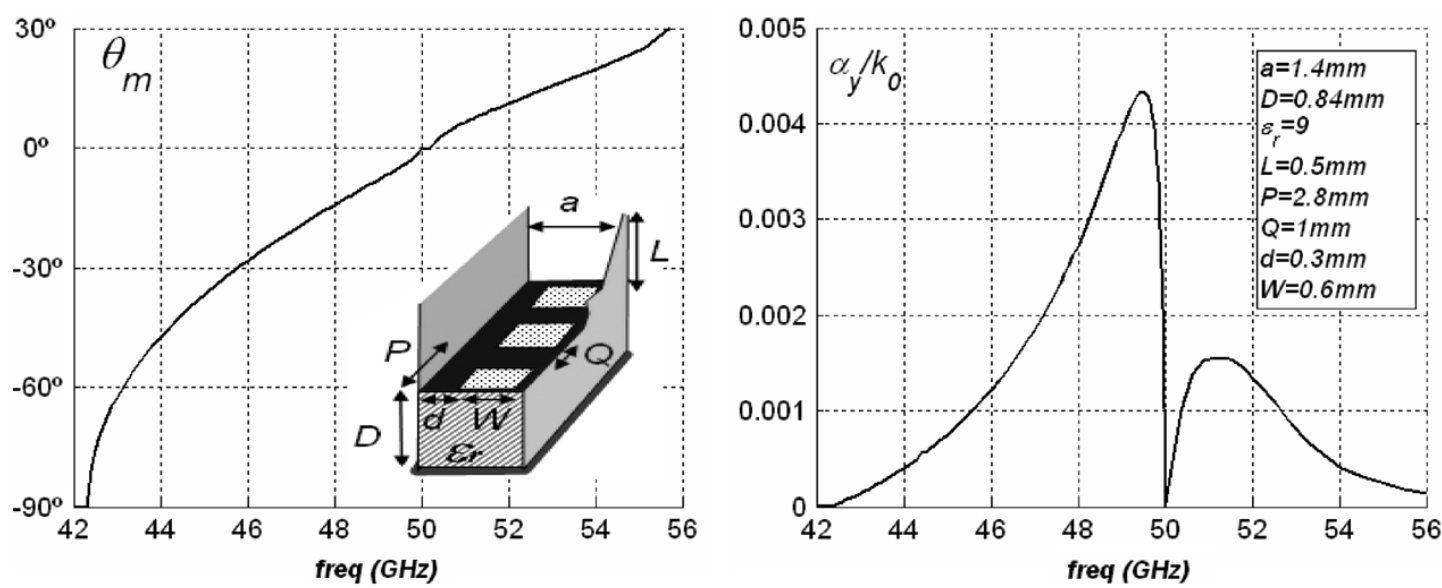

Fig. 8. Backward to forward frequency scanning capability of the new APLWA.
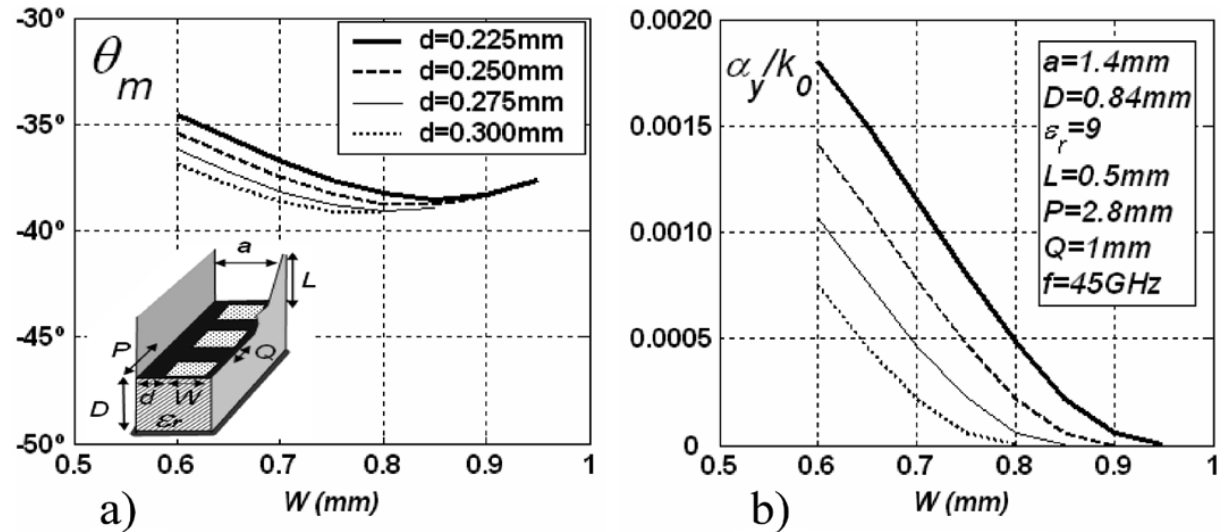

Fig. 9. Variation of the leakage constant by changing the slot width in the APLWA.

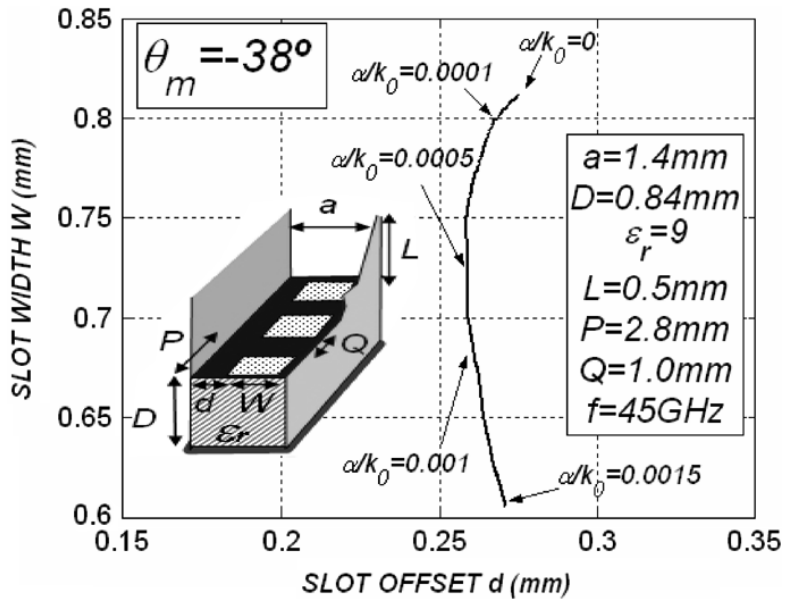

Fig. 10. Constant pointing direction contour line for the design of the tapered illumination.

width $W$ is the main responsible for the leakage variation, while the slot position $d$ is modified to readjust the pointing direction and keep it constant at $\theta_{m}=-38^{\circ}$ for all the values of $\alpha / k_{0}$. The aperture illumination determines the sidelobes level. To obtain a given illumination function $M(y)$, the leakage rate must be varied along the antenna longitudinal direction ( $y$ axis in
Fig. 1), according to the following known expression [2], where $L_{A}$ is the antenna length and $\eta$ is the antenna efficiency

$$
\alpha(y)=\frac{1}{2} \frac{|M(y)|^{2}}{\frac{1}{\eta} \cdot \int_{y=0}^{y=L_{A}}|M(y)|^{2} \cdot d y-\int_{y=0}^{y}|M(y)|^{2} \cdot d y} .
$$

\section{Radiation Patterns}

If the antenna is not tapered, the exponential illumination of the uniform leaky-mode creates a radiation pattern with $-13 \mathrm{~dB}$ sidelobes level. Fig. 11 shows the normalized radiation pattern obtained for the nontapered APLWA at $45 \mathrm{GHz}$, with the same dimensions as in Fig. 8, and using an antenna length $L_{A}=$ $10 \lambda_{0}$. The leaky-mode design predicts a pointing direction of $\theta_{m}=-38^{\circ}$, and a $-3 \mathrm{~dB}$ beamwidth of $\Delta \theta=9^{\circ}$. The radiation pattern has also been obtained using 3-D electromagnetic analysis software (HFSS), and it is plotted with circles in Fig. 11. The layout of the hybrid waveguide printed-circuit APLWA is shown in the inset of Fig. 11. The results obtained with HFSS agree very well with the radiation pattern obtained from the 2-D leaky-mode analysis, thus confirming the nontapered design.

To reduce the $-13 \mathrm{~dB}$ sidelobes level, a cosine-tapered illumination function $M(y)$ is chosen. Using (9) and the curve of Fig. 10, the variation of the periodic slots dimensions ( $W$ and 

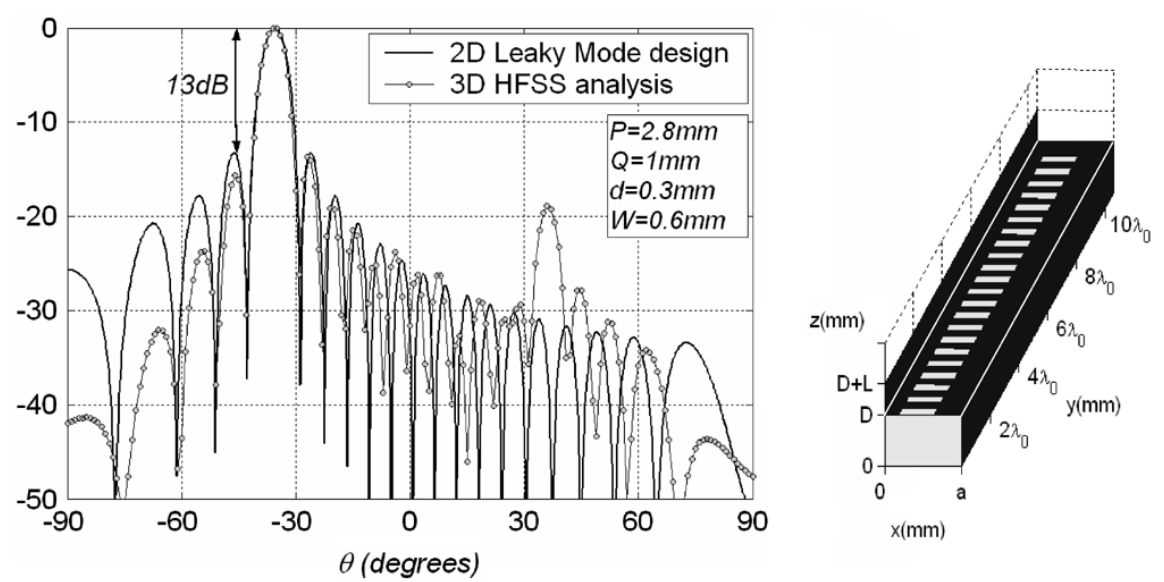

Fig. 11. Normalized radiation pattern for the nontapered APLWA at $45 \mathrm{GHz}$.
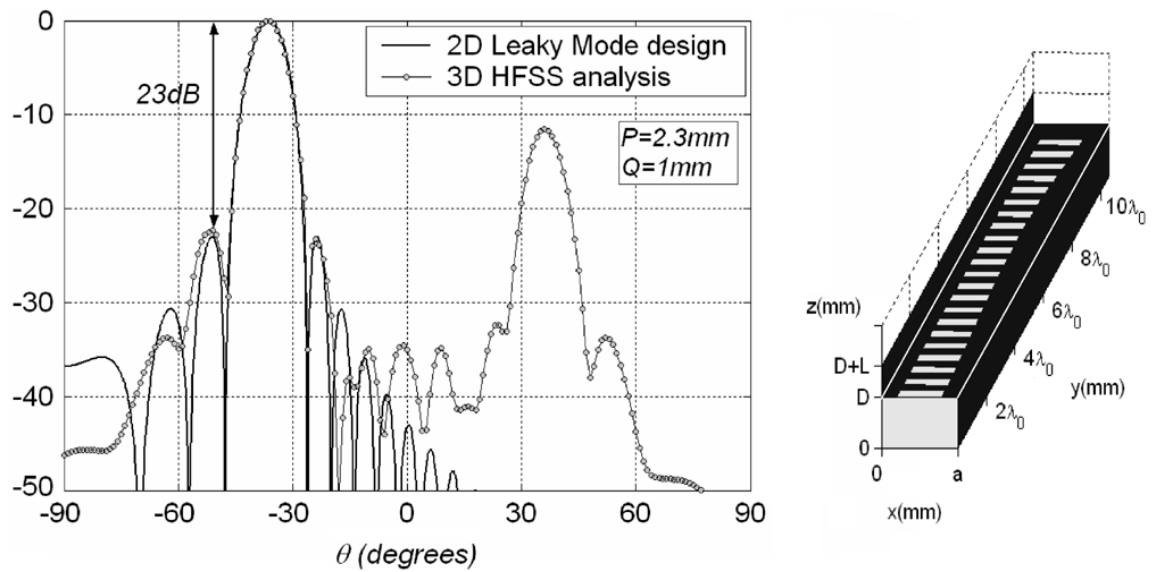

Fig. 12. Normalized radiation pattern for the tapered APLWA at $45 \mathrm{GHz}$.

d) along the antenna length can be designed to vary the leakage rate while keeping $\theta_{m}=-38^{\circ}$. Fig. 12 shows the layout obtained for the tapered APLWA, together with the normalized radiation pattern. It can be seen how the periodic slots width is modulated along the antenna length to create the cosine tapered illumination.

We also observe in Fig. 12 that the sidelobes level has been reduced to $23 \mathrm{~dB}$, as it corresponds to a cosine illumination. Again, the analysis of the 3-D antenna with HFSS agrees very well with the design performed using the leaky-mode 2-D analysis approach. There is, however, an important difference. The 3-D analysis takes into account the effect of the discontinuity at the end of the antenna length. A part of the leaky-mode energy is reflected back, creating a lobe in the forward quadrant, at the complementary angle of the main lobe $\left(\theta_{m}=+38^{\circ}\right)$. This reflected lobe is observed in both the tapered and the nontapered antennas (Figs. 12 and 11, respectively). In the nontapered antenna this effect is weaker due to the fact that a higher value of leakage rate was used $\left(\alpha / k_{0}=0.0015\right)$. For the tapered antenna, $\alpha / k_{0}$ must change from zero to the maximum affordable leakage rate [2], therefore having a lower mean leakage value (which corresponds to a greater reflected lobe). In any case, this unwanted lobe must be reduced below the sidelobes level in a practical tapered design. To reduce the reflected lobe, an improved transition has been added at the end of the antenna. The transition reduces the discontinuity from the periodically slitted structure to the completely closed waveguide. The scheme of such transition can be seen in the layout of Fig. 13. This transition is $2 \lambda_{0}$ long, making the antenna to be longer than in the previous design. However, this extra length does not affect the radiating length of the antenna, since the slots of the transition are centered and do not contribute to any radiation. Therefore, although the antenna total length is $12 \lambda_{0}$, the actual radiating length continues being $10 \lambda_{0}$. The radiation pattern obtained with HFSS shows an improvement in the reflected lobe, which has been reduced more than 10 $\mathrm{dB}$ with respect to the design without transition (Fig. 12). In this way, the cosine tapered illumination is not spoilt by the reflected lobe, since all the sidelobes are at least $23 \mathrm{~dB}$ below from the main lobe.

It must be noticed that the design has been performed using the results from the novel leaky-mode analysis technique proposed in this paper. This approach allows for fast computations of the leaky-mode dispersion curves, due to its high analytical 2-D analysis nature. Only the unit-cell antenna cross section is needed to obtain the leaky-mode complex propagation constant. Once the parametric behavior of the leaky-mode is known (with frequency and antenna dimensions), the tapered slot dimensions have been straightforwardly obtained to design a given illumination function. The design of the 3-D tapered antenna is there- 

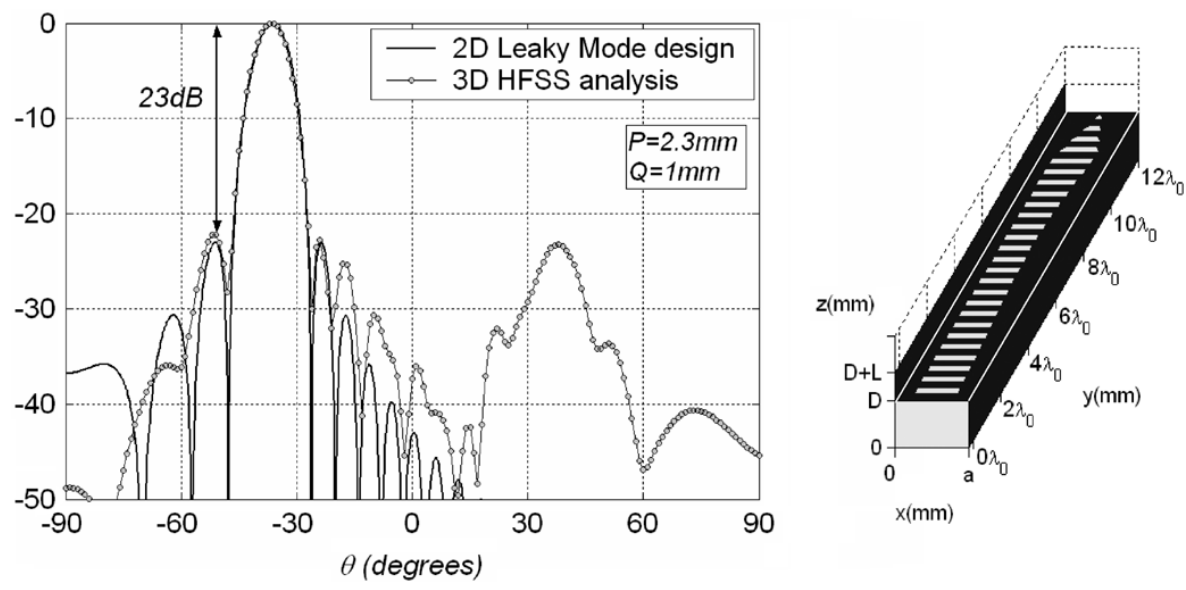

Fig. 13. Normalized radiation pattern for the tapered APLWA with transition from periodically slitted waveguide to closed waveguide to reduce the reflected lobe.

fore obtained from a 2-D analysis. The designed 3-D tapered-antenna structure has been validated using HFSS. The automated design would have been extremely time consuming using a 3-D analysis tool, due to the complexity and electrical length of the antenna (22 slots in a $10 \lambda_{0}$ antenna, each one with its own width and offset).

\section{CONCLUSION}

A full-wave spectral-domain technique has been specifically developed for the analysis of leaky-modes in laterally-shielded stub-loaded rectangular dielectric waveguides, periodically perturbed with rectangular strips or slots. The electromagnetic fields have been developed by means of Floquet and parallel-plate modes. The method of moments has been applied to solve the corresponding EFIE/MFIE for the periodic rectangular strip or slot discontinuities. Comparisons with previously studied leaky-wave modes in 1-D structures - both uniform and periodic-, have been presented, showing very good agreement. The full-wave feature of the proposed leaky-mode analysis method, together with its high analytical nature, allow to easily obtain the leaky-mode dispersion curves necessary for the design of a leaky-wave antenna. As an example, a novel PLWA has been presented and designed. This antenna has the same advantages that the dielectric-inset PLWA, which was presented a decade ago for applications in the millimeter waveband. In addition, the new antenna presents the property to independently control the stopband width and the leaky-mode leakage rate. This is due to the fact that the new antenna uses the asymmetry radiation principle, therefore being called the APLWA. In this way, one can design a low-sidelobes tapered-illumination antenna, while keeping the ability to scan from the backward to the forward quadrant with a narrow stopband. The leaky-mode design has been compared with 3-D electromagnetic analysis using commercial software to validate a cosine-tapered antenna radiating in the backward quadrant.

\section{ACKNOWLEDGMENT}

The authors would like to thank the EPSON-Ibérica Foundation for its stimulating support.

\section{REFERENCES}

[1] R. E. Collins and F. J. Zucker, Antenna Theory, Part 2. New York: McGraw-Hill, 1969, ch. 19-20.

[2] A. A. Oliner and R. C. Johnson, Leaky-Wave Antennas, Antenna Engineering Handbook, 3rd ed. New York: McGraw-Hill, 1993, ch. 10.

[3] M. Ghomi and H. Baudrand, "Full-wave analysis of microstrip leakywave antenna," Elect. Lett., vol. 25, no. 13, pp. 870-871, Jun. 1989.

[4] C.-K. C. Tzuang and Y.-C. Chen, "Dispersion characteristics of microstrip with periodic perturbations," in Proc. IEEE Microwave Int. Symp. Dig., vol. 3, 2000, pp. 1537-1540.

[5] F. Schwering and S. T. Peng, "Design of dielectric grating antennas for millimeter-wave applications," IEEE Trans. Microw. Theory Tech., vol. 31, pp. 199-209, Feb. 1983.

[6] J. Jacobsen, "Analytical, numerical and experimental investigation of guided waves on a periodically strip-loaded dielectric Slab," IEEE Trans. Antennas Propag., vol. AP-18, no. 3, pp. 379-387, May 1970.

[7] M. Mittra and R. Kastner, "A Spectral domain approach for computing the radiation characteristics of a leaky-wave antenna for millimeter waves," IEEE Trans. Antennas Propag., vol. AP-29, no. 4, pp. 652-654, Jul. 1981.

[8] H. A. Kalhor, "Electromagnetic scattering by a dielectric slab loaded with a periodic array of strips over a ground plane," IEEE Trans. Antennas Propag., vol. 36, no. 1, pp. 147-151, Jan. 1988.

[9] J. A. Encinar, "Mode-matching and point-matching techniques applied to the analysis of metal-strip-loaded dielectric antennas," IEEE Trans. Antennas Propag., vol. 38, no. 9, pp. 1405-1412, Sep. 1990.

[10] T. Rozzi and L. Ma, "Scattering by dipoles in inset dielectric guide and application to millimetric leaky wave antennas," in Proc. 17th Eur. Microwave Conf., Rome, Italy, Sep. 1987, pp. 543-548.

[11] M. Guglielmi and H. Hochstadt, "Multimode network description of a planar periodic metal-strip grating at a dielectric interface: Part III-Rigorous solution," IEEE Trans. Microwav. Theory Tech., vol. 37, no. 5, pp. 902-909, May 1989.

[12] M. Guglielmi and G. Boccalone, "A novel theory for dielectric-inset waveguide leaky-wave antennas," IEEE Trans. Antennas Propag., vol. 39, no. 4, pp. 497-504, Apr. 1991.

[13] J. L. Gómez, A. de la Torre, D. Cañete, M. Gugliemi, and A. A. Melcón, "Design of tapered leaky-wave antennas in hybrid waveguide-planar technology for millimeter waveband applications," IEEE Trans. Antennas Propag.: Special Issue on Antennas Propag. Applicat., pt. I, vol. 53, no. 7, pp. 2563-2577, Jul. 2005.

[14] J. L. Gómez and A. A. Melcón, "Nonorthogonality relations between complex-hybrid-modes: An application for the leaky-wave analysis of laterally-shielded top-open planar transmission lines," IEEE Trans. Microwave Theory Tech., vol. 52, no. 3, pp. 760-767, Mar. 2004.

[15] J. L. Gómez and A. A. Melcón, "Radiation analysis in the space domain of laterally-shielded planar transmission lines. Part I: Theory," Radio Sci., vol. 39, no. 3, pp. 1-11, Jun. 2004.

[16] F. J. Glandorf and I. Wolff, "A spectral-domain analysis of periodically nonuniform microstrip lines," IEEE Trans. Microw. Theory Tech., vol. MTT-35, no. 3, pp. 336-343, Mar. 1987.

[17] K. Wu, V. Dzougaiev, and P. Saguet, "Complete theoretical and experimental analysis on properties of planar periodic waveguides," Proc. Inst. Elect. Eng., vol. 135, pp. 27-33, Feb. 1988. 
[18] P. Baccarelli, P. Burghignoli, C. Di Nallo, F. Frezza, A. Galli, P. Lampariello, and G. Ruggieri, "Full-wave analysis of printed leaky-wave phase arrays," RF and Microw. Comput. Aided Eng., vol. 12, no. 3, pp. 272-285, May 2002.

[19] P. Lampariello and A. A. Oliner, "A new leaky-wave antenna for millimeter waves using an asymmetric strip in groove guide: Part I-Theory," IEEE Trans. Antennas Propag., vol. 33, no. 12, pp. 1285-1294, Dec. 1985.

[20] P. Lampariello and A. A. Oliner, "A novel phase array of printed-circuit leaky wave lines sources," in Proc. 17th Eur. Microwave Conf., Rome, Italy, 1987, pp. 555-560.

[21] M. Guglielmi and A. A. Oliner, "Multimode network description of a planar periodic metal-strip grating at a dielectric interface. Part II: Smallaperture and small-obstacle approximations," IEEE Trans. Microwave Theory Tech., vol. 37, no. 3, pp. 542-551, Mar. 1989.

[22] M. Guglielmi and D. R. Jackson, "Broadside radiation from periodic leaky-wave antennas," IEEE Trans. Antennas Propag., vol. 41, no. 1, pp. 31-37, Jan. 1993.

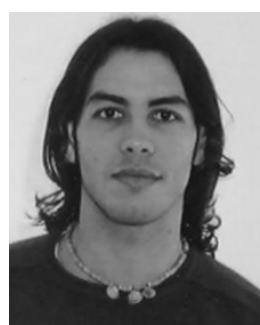

José Luis Gómez Tornero was born in Murcia, Spain, in 1977. He received the telecommunications engineer degree from the Polytechnic University of Valencia (UPV), Valencia, Spain, in 2001, and the Ph.D. degree (laurea cum laude) in telecommunication engineering from the Technical University of Cartagena (UPCT), Cartagena, Spain, in 2005.

In 1999, he joined the Radiocommunications Department, UPV, as a Research Student, where he was involved in the development of analytical and numerical tools for the study and automated design of microwave filters in waveguide technology for space applications. In 2000, he joined the Radio Frequency Division, Industry Alcatel Espacio, Madrid, Spain, where he was involved with the development of microwave active circuits for telemetry, tracking and control (TTC) transponders implicated in many different spatial missions for the European Space Agency (ESA), National Aeronautics Space Administration (NASA) and other Space Agencies. In 2001, he joined the Technical University of Cartagena, Spain, as an Assistant Professor, where he is currently developing his teaching activities. His scientific research is focused on the analysis and design of LWA for millimeter wave-band applications and the development of numerical methods for the analysis of novel passive radiating structures in planar and waveguide technologies. His scientific interests also include the study of active devices for microwave and millimeter wavebands, such as oscillators and active antennas.

Prof. Gómez Tornero received the Second National Award from the EPSONIbérica Foundation for the best Ph.D. project in the field of technology of information and communications (TIC) in July 2004.

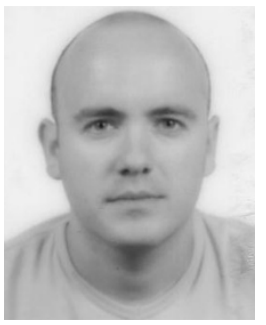

Fernando Daniel Quesada Pereira was born in Murcia, Spain, in 1974. He received the telecommunications engineer degree from the Polytechnic University of Valencia (UPV), Valencia, Spain, in 2000. He is currently working toward the Ph.D. degree at the Technical University of Cartagena (UPCT), Cartagena, Spain.

In 1999, he joined the Radiocommunications Department, UPV, as a Research Assistant, where he was involved in the development of numerical methods for the analysis of anechoic chambers and tag antennas. In 2001, he joined the UPCT, first as a Research Assistant and then as an Assistant Professor. His current scientific interests are the integral equation numerical methods for the analysis of antennas and microwave devices.

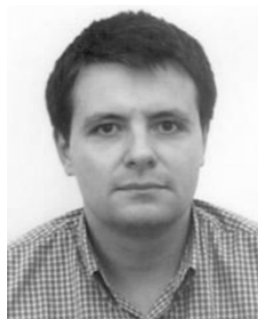

Alejandro Alvarez Melcón (M’98) was born in Madrid, Spain, in 1965. He received the telecommunications engineer degree from the Polytechnic University of Madrid (UPM), Madrid, Spain, in 1991, and the Ph.D. degree in electrical engineering from the Swiss Federal Institute of Technology, Lausanne, Switzerland, in 1998.

In 1988, he joined the Signal, Systems and Radiocommunications Department, UPM, as a Research Student, where he was involved in the design, testing, and measurement of broad-band spiral antennas for electromagnetic measurements support (EMS) equipment. From 1991 to 1993, he joined the Radio Frequency Systems Division, European Space Agency (ESA/ESTEC), Noordwijk, The Netherlands, where he was involved in the development of analytical and numerical tools for the study of waveguide discontinuities, planar transmission lines, and microwave filters. From 1993 to 1995, he joined the Space Division, Industry Alcatel Espacio, Madrid, Spain, and he worked at the ESA, where he collaborated in several ESA/ESTEC contracts. From 1995 to 1999, he was with the Swiss Federal Institute of Technology, École Polytechnique Fédérale de Lausanne, Lausanne, Switzerland, where he worked in the field of microstrip antennas and printed circuits for space applications. In 2000, he joined the Technical University of Cartagena, Spain, where he is currently developing his teaching and research activities.

Dr. Álvarez Melcón received the Journée Internationales de Nice sur les Antennes (JINA) Best Paper Award for the best contribution to the JINA'98 International Symposium on Antennas, and the COIT/AEIT (Colegio Oficial de Ingenieros de Telecomunicación) award to the best Ph.D. thesis in basic information and communication technologies. 